\title{
Adaptation of Information Quality and Assurance Management Paradigm in a Strategic Public Sector Organization
}

\author{
SHAHZAD NAEEM*, AND MUHAMMAD HASAN ISLAM* \\ RECEIVED ON 25.02.2015 ACCEPTED ON 16.09.2015
}

\begin{abstract}
Information quality despite being a critical area in organizations lacks in comprehensive methodologies for its adaptation and improvement even after years of active research and practice. Therefore adaptation of information quality while ensuring information assurance has become a major concern for strategic organizations to upgrade their IT (Information Technology) Assets. The issue exists specifically in organizations with integrated access to distributed data. In this article, a new model is proposed that is derived after addressing issues in already existing information quality and information assurance adaptation models, showing the methodology and its advantages for strategic organizations. However, this model has to be adapted to the requirements and needs of an organization; since it is a reference model. The steps and procedures are identified in quality and assurance adaptation model to bring abstract standard into practice.
\end{abstract}

Key Words: Data Strategy, Information Assurance, Information Quality, Strategic Organization, Quality Assurance, Quality Adaptation.

\section{INTRODUCTION}

$\mathrm{I}$

n today's era, information data produced and stored by organizations is far greater than ever before. Subsequently, excess of information along with insufficient management of information quality has resulted many organizations into leading to an uncertain situation in information quality and assurance. In addition, evaluation, development and management of data has proved to be very difficult to perform. Information quality management in organization is a mission success essential factor. Information quality management certifies availability of correct data at the right time to the right individual helps make an effective decision making process. An organization that has long term effects on the stature and well-being of society can be termed as strategic organization. These organizations may well include public sector organizations such as, health department, education commissions, armed forces of a country and poverty alleviation departments. In a strategic organization, information quality and assurance management process is a combination of regulations and measures taken to guarantee that data information is fulfilling the quality and assurance metrics for effective utilization in Command and Control, logistics and other support services that enable mission promptness, trustworthiness and efficiency. Moreover, cost of automated support to a strategic organization is reduced by improvement in information quality resulted by rationalizing management and technical data. In modern

* College of Electrical \& Mechanical Engineering, National University of Science \& Technology, Rawalpindi

Mehran University Research Journal of Engineering \& Technology, Volume 35, No. 3, July, 2016 [p-ISSN: 0254-7821, e-ISSN: 2413-7219] 
data architectures, organization's data resides in its own data centres as well as distributed across remote locations or at public and private clouds. Hybrid cloud architectures provides considerable major economic advantages, yet their implementation demands a more mature and experienced approach to information assurance; this will include integrated monitoring and management capabilities as well as information security management at the level of each individual digital asset. Models for adaptation and implementation of information quality and assurance exist but all pose certain limitations in application to different sets of organizations. This paper will provide a comprehensive information quality and assurance adaptation and implementation model for strategic organizations.

The purpose of this study is to analyze issues in existing information quality models and propose a model that addresses all the current issues while ensuring security in any government organization with strategic objectives.

The rest of this paper is organized as follows. In Section 2, we provide a brief literature review, followed by considerations, issues involved in formulating an information quality and assurance strategy in Section 3. Section 4 proposes a Data quality and assurance model that addresses the issues outlined in section 3 . Section 5 presents the implementation results of proposed model in two public sector strategic organizations and Section 6 discusses the findings. Section 7 concludes the paper by summarizing the work done and provides research direction for further work.

\section{RESEARCH OBJECTIVES}

The notion of information quality adaptation model includes different aspects organizational operations and quality information classifications. The fact remains is that there does not exist a model of information quality adaptation that can address the existing issues in existing models and ensuring information security perspectives. In this paper an attempt is being made to propose an information quality adaptation model designed to focus on strategic organizations of all types. The model will ensure resolving the existing problems in existing major industry adopted models with an added feature of address assuring information security at each and every individual level.

\section{LITERATURE REVIEW}

Literature demonstrates that numerous efforts have been made to identify critical factors that lead to success and the amount of information quality management required alongside the quality management [1]. Conversely, identifying the success factors in AIS (Automated Information Systems) remains unspecified and has thus resulted into limited research efforts of critical success factors in the area.

According to Dravis [2] Strategy can be defined as a group of decisions aimed at the goals and objectives to find in what way resources should be utilized and actions be taken. The best utilization of data and level of quality and assurance required for the purpose will be determined by organizational goals. Actions are determined as procedures that are rectified and triggered for data management. The same definition of strategy can be rephrased for application as information quality definition, as a group of decisions aimed at the information quality goals and objectives of an organization to find in what way manpower should be utilized and actions be taken and solutions to implement. There is no consensus about terminology and methods of quality because quality is determined from different viewpoints and aspects [3]. According to Ehlers [4] quality is a multi-front concept with many interpretations. A definition of quality proposed by Juran [5] that is used commonly is "fitness for purpose". Similar to "Strategy", Quality is also dependent on goals and objectives of an organization.

The concept of TQM (Total Quality Management) has been implemented in certain sectors for generic purposes, such as, higher education management [6], information 
systems management [7] and software development [8]. On the other hand, for certain precise purposes many other models have been proposed, for example, for information quality metrics [9] and for education and training performance [10].

The main idea behind maintaining strategic information quality as discussed by Coleman [11] is that such an organization is strictly required to identify that in what manner its data uses and data itself will change over time. As data of an organization affects all of its facets and at the same time with passage of time uses of data change over time, therefore, maintaining data with high-quality demands a total and wholesome emphasis on producing good data. Issues associated with information quality can cause harm to an organization in form of inadequate information assurance measures that may range from losing user's confidence and customer's mistrust to loss of mission and even human life. This leaves that, in a strategic organization information quality management along with information assurance are among key mandatory requirements for its mission success.

The information quality and assurance adaptation model is mainly an issue that roots to continuous management supervision and monitoring to measure and analyze data through assessments, verification and validation. Experts in quality control methodology [12] always recommend addressing the "root cause". Anticipated information quality metrics including accuracy, completeness, timeliness, consistency, conformity and record duplication and anticipated information assurance metrics including, confidentiality, integrity, authentication, non-repudiation of data should be considered while addressing the root causes of information quality and assurance.

\section{ISSUES IN EXISTING INFORMATION QUALITY AND ASSURANCE MODELS}

The quality of information has transformed into a major apprehension for strategic organizations and a major area of research in Management Information Systems. In organizations, the demand for and awareness of high quality information has increased growth of data warehouses and access to data from different sources for administrators and higher management. In Data Quality model presented by Johns [13], more focus has been made on health care data. The health care industry data models are technically strategic in nature as such data do not require change over a longer period of time. Therefore, health data models cannot be equally applied to other organizations with strategic objectives. Changes and modifications in health care information systems do not require changes in the data that is classified to be quality data. Insufficient or incomplete knowledge and understanding of TQM, lack of commitment by management, incompetent teamwork and incapability to modify or alter organizational culture are main steps to be addressed and overcome for successful quality management [14]. Another model - Quality adaptation model by Pawlowski [3] proposes that four main steps are necessary to successfully adapt and implement an information quality model: context setting, model adaptation, model implementation/adoption, and quality development, where each step should be performed with a broad range of actors to raise awareness and consensus. The model remains comprehensive solution for implementation in a small scale strategic organization, but for adoption in a large scale public sector organization it lacks both verification and validation of processes involved. In addition, Pawlowski's [3] model also limits in continuous monitoring at all stages. On the other hand, TDQM model [15] proposed by US DoD (Department of Defense) provides for the evaluation of information quality management process. The idea is to review information quality goals/benefits and to improve processes used to manage information quality. The model remains applicable to public sector strategic organization but poses limitations in application to organization that requires adaptation and implementation of new information quality system. On the other hand, information assurance model 
frameworks [16-17] lacks addressing the information quality concerns in their models by only focusing the information assurance goals and objectives.

Based on the analysis discussed regarding existing information quality models, it can be concluded that the system clearly lacks availability of a comprehensive model that can be used to implement information quality in a public sector organization with strategic goals.

Further to the issues discussed in the previous paragraph, some additional information quality and assurance areas of concern are lack of continuous monitoring of the whole adaptation process and addressing the root causes of information quality and assurance issues.

Continuous Monitoring: Includes measuring, analyzing, and then improving a system at each individual phase while adhering to the principals of TQM which is also required to effectively confront performance issues.

In US DoD [15] research, it is mentioned that root causes behind problems in information quality will always be triggered by one of the following areas.

\subsection{System Problem}

Problems in information quality mainly strengthen due to limitations in system design and aided by improper modifications in documentation with partial or no training of users or caused by the systems that lacks scalability.

\subsection{Policy and Procedure Problem}

Another reason of information quality problems can be either contradicting guidance in present policies and SOPs (Standard of Procedure), failure to follow the present policies and SOPs or deficient in suitable guidance.

\subsection{Data Design Problem}

There exists a possibility that the data errors are allowed into the system by database itself due to batch loads, improper user privilege specifications and use of incomplete data constraints.
In addition to above mentioned root causes of information quality, another area of grave concern in modern era for public sector strategic organizations is Information Assurance in all project plans that involve architecture modification.

\subsection{Lack of Information Assurance Capabilities}

Existing models of information quality do not address security concerns and typically Information Assurance concerns are ignored to achieve better performance while implementing.

\section{PROPOSED MODEL - HYBRID INFORMATION AND QUALITY ASSURANCE}

The proposed information quality assurance model is derived after addressing the issues in existing information assurance and quality assurance models discussed in previous section while addressing limitations of each as well as focusing on the information assurance objectives, which makes it a more comprehensive and complete for implementation in a strategic organization. In this context, Adaptation indicates that reference model proposed can only serve as a guideline upon which phases should be built. In addition, proposed HIQA (Hybrid Information and Quality Assurance) model recommends certain measures to overcome the obstructions in quality management that includes incomplete/ insufficient knowledge of the system and lack of management commitment.

Additionally, the model suggests steps to overcome the main barriers of quality management, such as the lack of management commitment and inadequate knowledge. Our proposed model broadly addresses the following areas:

Application to strategic organization with broader goals and objectives including both information quality and information assurance. 
Root causes of data quality problems are addressed.

- Monitoring process throughout for achievement of desired goals and achievement of assurance objectives.

Adaptation in a strategic organization.

The proposed stages of adaptation of data quality are outlined in Fig. 1. It begins with formulation of a data strategy by creating system awareness, and requirements identification, its implementation followed by verification and validation to ensure meeting objectives. The model also encompasses evaluation of achieved output. All the phases proposed in this model will include addressing primary root causes of data quality issues outline in previous section as well as the process of continuous monitoring will be executed in parallel.

The activities involved in these phases are further elaborated and explained as follows.

\subsection{Data Strategy}

As first step in the HIQA model process, establishing the right secure environment for information quality management is supposed to be among the most challenging steps in the process. Creating information quality and assurance environment will include involvement of both information system administrators and functional users. Fig. 2 depicts Data Strategy broadly.

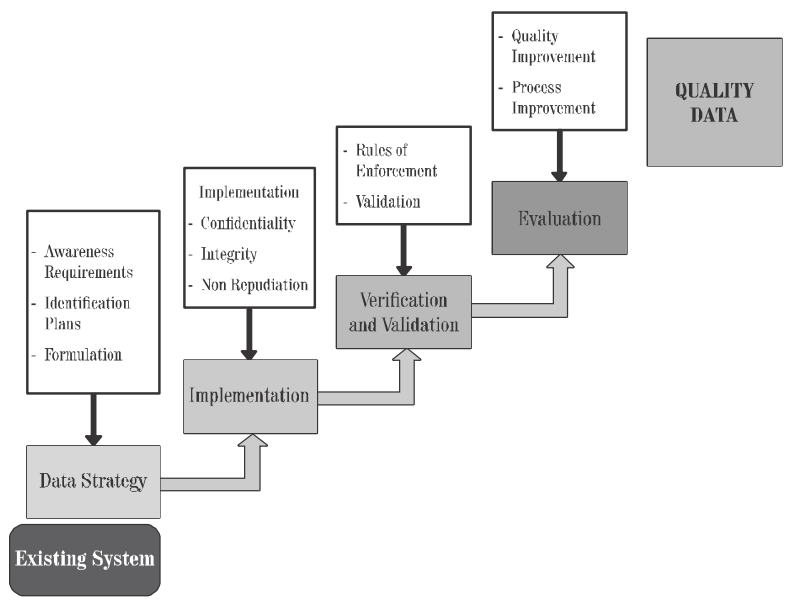

FIG. 1. PROPOSED HIQA FRAMEWORK

\subsubsection{Awareness}

The vision, strategy, and policy statements are mainly the constituents of an organization's long term or strategic objectives. Awareness among the top management of organization, its functional users and automated Information System administrators requires the redefinition of organization's vision, strategies and policies. The process, that is setting fresh course for organizational commitments, should be preferably familiar to all the stakeholders. As for example, strategy/policy should explain how changes and modifications in information management correlate to organization's fundamental operations and capabilities. Thus process of awarenessraising is a directly related. All staff of establishment must have familiarity with quality and accordance of their actions with it.

\subsubsection{Requirements Identification}

The success factors for quality should be defined by building confidence and consensus among all stakeholders. All requirements should be defined in this phase; this includes requirements that may be classified as quality data and identifying the information assurance measures to be implemented in the system keeping in view any system changes and modifications and objectives and goals of organization.

\subsubsection{Plans Formulation}

Implementation plans shall be formulated from the viewpoint of project management and focused on following:

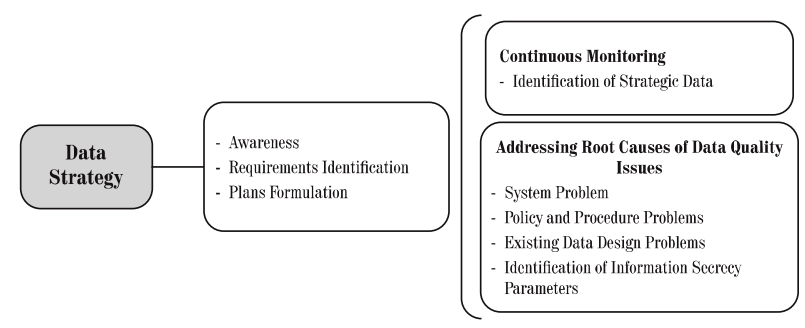

FIG. 2. DATA STATAGY (PHASE-1)

Mehran University Research Journal of Engineering \& Technology, Volume 35, No. 3, July, 2016 [p-ISSN: 0254-7821, e-ISSN: 2413-7219] 
Assignment Brief: Lists project goals, scope and outline of anticipated outcomes.

Assignment Description: It will include tasks related to information quality.

Project Line: Briefly outline the tasks to be completed and tools to be used and measures to be taken for both of these forming baselines for information quality.

Schedule: Determines projects timelines and milestones.

Deliverables: Maintain a record of reports and products that are used for documentation of the outcomes of information quality project. This should also include a technical after-action-report on information quality which outlines enhancements in information quality achieved and the shortcomings to overcome.

Resources: It will include among other direct and indirect costs, costs in relation to preparation, tools and manpower utilization.

\subsection{Implementation}

Usually small actors are involved in making the concept successful in the initial stages of implementing an adaptation process. This increases importance of formulation of a comprehensive implementation strategy with robust security measures outlining activities and actions of quality system. According to Thiagarajan and Zairi [18] involvement of all the stakeholders in the quality adaptation process is of vital importance. Also it is pertinent to mention that, familiarity with information and quality assurance system by all stakeholders does not necessarily require all staff members in order to maintain the secrecy of classified information functions. After completion of the implementation phase (Fig. 3), a comprehensive verification and validation test will be conducted to quantify and qualify the outcomes.

\subsection{Verification and Validation}

In verification phase, the process is assessed for correctness of data, its completeness and conformance to defined procedures or predetermined requirements. Documenting after ensuring that output data is what it is supposed to be is the primary aim of verification. It verifies the correctness of data output with focus on achievement of preset security goals.

The validation phase is primarily aimed to determine the analytical quality of a specific data set. These validation measurements focus on the main Quality Assurance plans and objectives and to determine whether the associated security measures are implemented correctly. An individual(s) who is not directly or indirectly part of the activity being performed should primarily be performing the validation phase. Broader objectives of this phase are mentioned in Fig. 4.

\subsection{Evaluation}

The last step of HIQA, evaluation and assessment of any improvements/progress is carried out that is achieved during the whole project. These improvements will be

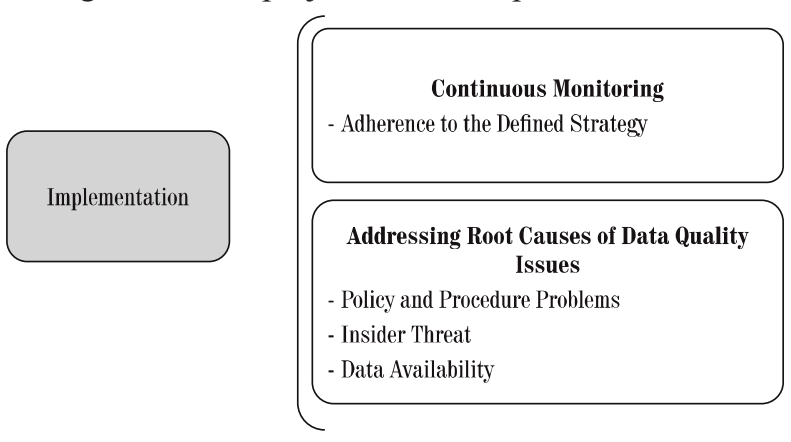

FIG. 3. IMPLEMENTATION (PHASE-II)

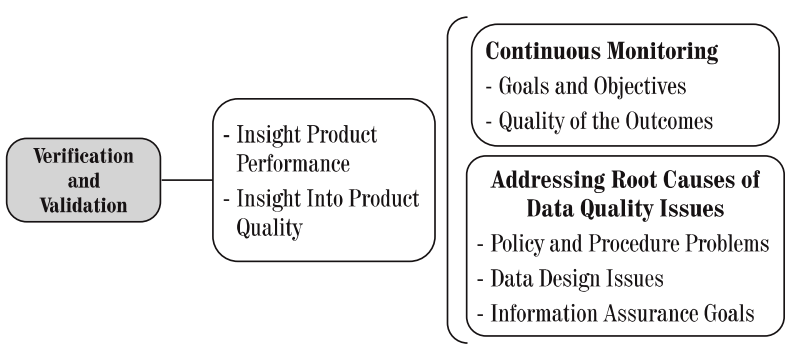

FIG. 4. VERIFICATION AND VALIDATION (PHASE-III)

Mehran University Research Journal of Engineering \& Technology, Volume 35, No. 3, July, 2016 [p-ISSN: 0254-7821, e-ISSN: 2413-7219] 
examined with respect to: (1) modifying or current methodology to adapt data quality management and/or (2) find whether or not the project has led to any achievements and accomplishments of its goals and objectives (3) Information Assurance parameters set at the start of process are met or not. The considerations of this step are mentioned in Fig. 5.

A quality system demands high requirement of continuous evaluation, updating, and enhancements in conjunction to the novel developments in a strategic organization. The information quality will be evaluated to find whether or not the quality adaptation system has guided to overall improvements in organization's performance. The evaluation phase also includes assessment of quality metrics and any improvement actions required at a different point of time as for example in system design modification. The outcome of this phase is an evaluation strategy, improvement concepts, and, most important, a broad discourse on quality.

The pyramid shown in Fig. 6 shows complete proposed model that includes continuous monitoring and addressing main root causes of issues in data quality models at each individual process phase. This also remains a distinguishing characteristic of proposed model.

\section{ANALYSIS OF HIQA MODEL}

The methodology used to analyze efficiency of model is Action Research, in which links practice and theory to solve problems that are more of a practical nature [19][20]. Kemmis and Mctaggart [21] developed one of

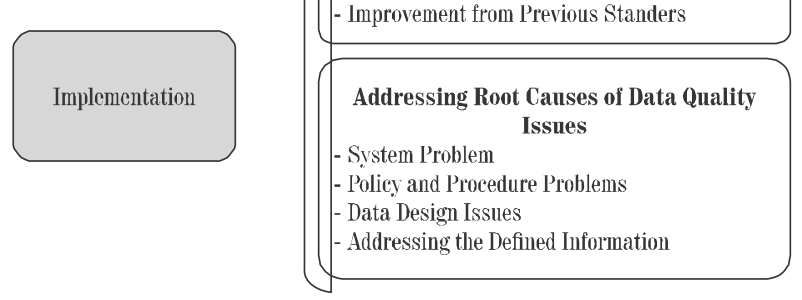

FIG. 5. EVALUATION (PHASE-IV) the most widely used approaches to action research. Each action research cycle consists of the following steps (Fig. 7):

- Plan: Formulate a plan of action aiming to increase efficiency of current practices.

- Act: The members responsible for implementing the plan act mutually to execute the plan.

- Observe: Through evaluation of outcomes, the actions are observed to collect evidence.

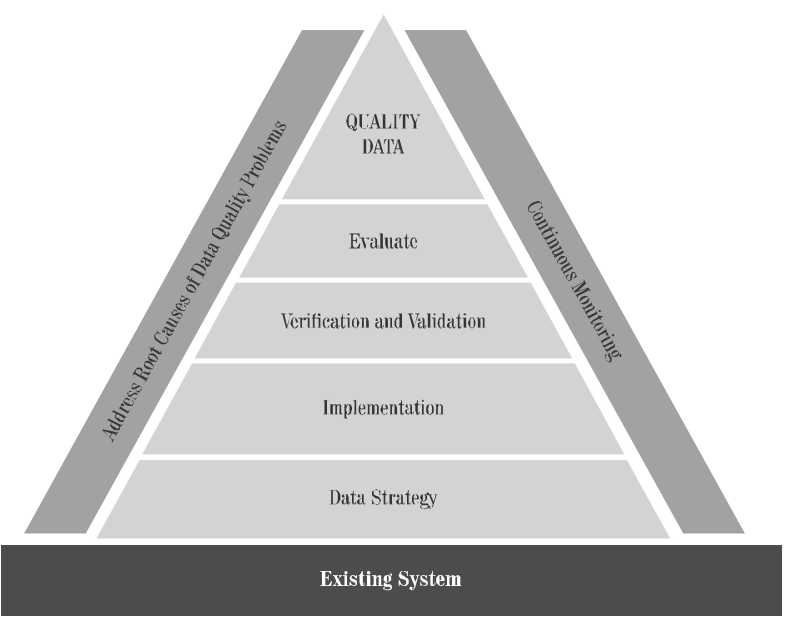

FIG. 6. COMPLETE HIQA FRAMEWORK

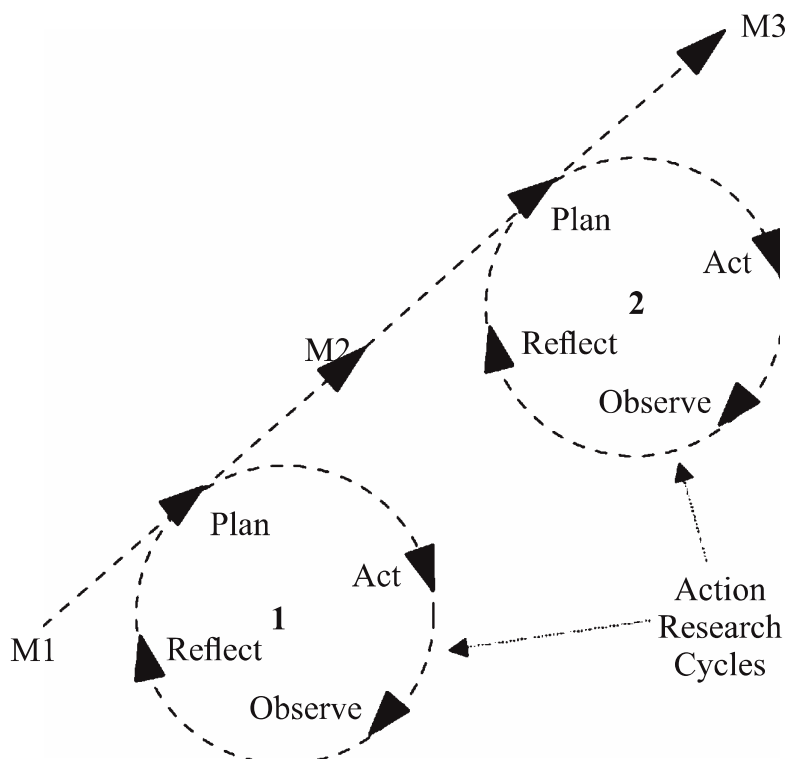

FIG. 7. ACTION RESEARCH CYCLE 
Reflect: The member participants give feedback on what was incorrect, what remained right and proposition to improve the idea in the next cycle. Each cycle may guide to perfection in the initial logic (M1), this will lead to more refined ideas in a row. M2, M3 etc.

\subsection{Justification for Research Method Selection}

At this stage of research, Action research is chosen to be a suitable research method for following main reasons:

- It is a field based method, which allows evaluation of metrics "in an organizational context using real practitioners", as recommended in [22].

- It is a qualitative method, and is therefore suitable for exploratory research [22].

- Cost of the implementation of this model is supposed to be high as at every stage it contains continuous monitoring, but keeping in view the nature and objectives of strategic organization, it cannot be ignored.

\subsection{Organizational Context}

Action research was conducted in two public sector strategic organizations where the model was completely implemented. Main stakeholders identified in the action research included indirect users who were project modeling analysts that are accountable for data model development.

\section{FINDINGS}

Instead of reporting the results of each action research phase, the results are summarized as the cycles were conducted in a cumulative approach.

\subsection{Number of Phases}

In both government organizations, there were only a limited number of people qualified or available to conduct reviews, and the need to calculate the value of each phase added significantly to their workload. A basic philosophical difference is highlighted at this point, differentiating practice from research:

- Research tends to strive for completeness and closure - in this case, to measure all possible aspects of quality.

Practice tends to focus on what is necessary to get the job done - To measure only those aspects that are most important for improving quality an "80/20" approach. This reflects a focus on utility.

The need for completeness in measuring information model quality must be balanced with need for the measurement approach to be practical and useable.

\subsection{Usage of Phases}

The reviewers while implementing model expressed their concern over how exactly phases of model should be interpreted. However a productive outcome of study was to explain how the stages could be used. Mainly, following possible uses of each phase was identified:

To identify the most efficient option among alternate models available (model choice)

To enhance the model quality. (product quality)

To carryout comparison of different projects and to improve the process of data modeling over time (process quality).

\subsection{Use of Phases is a Cost-Benefit}

Quality adaptation and adoption is always costly and is deployed if the perceived benefits outweigh the efforts required to deploy them [23]. However, in a public sector strategic organization, dimensions of judgment and reasoning are different. Thus, discussions with participants during reflect phases of action research showed that their perceptions of usefulness of a particular phase and their consequent willingness to use it are a pragmatic decision based on: 
Cost: Perceived cost or effort required at each phase (reviewer's viewpoint)

Benefit: Perceived benefits in terms of improving the quality of information (reviewer and reviewer's viewpoint)

\subsection{Validation of Phase}

In a public sector strategic organization, it is not affordable to take chances for greater prosperity of public associated with the organization and for this reason cost of availing quality is ignored keeping in view the outcome. It was commonly expressed by reviewers that, following phases of model is a generic process to achieve information quality and assurance but to ensure its validation for a longer period of time to ensure its strategic nature it is highly suitable to conduct the following two operations in each phase.

Addressing the root causes of Information Quality and Information Assurance

Continuous monitoring for achievement of desired goals and objectives of organization.

The proposed model can equally be applied in organizations that include public sector organizations such as, health department, education commissions, armed forces of a country and poverty alleviation departments.

As discussed earlier, implementation of information quality and assurance adaptation process and achievement of information quality in strategic organization requires ignoring cost of implementation, thus making the achievement of objectives more feasible. The results achieved would not have been possible otherwise by implementing the process in a laboratory.

\section{CONCLUSIONS}

In this paper, suitability of current quality and assurance standards in practice and their use in different organizations were discussed. To implement a comprehensive information quality and assurance system in a strategic organization, major phases involved are: formulating a data strategy, its implementation, verification and validation of the process and evaluation of the overall output. All phases of the model will be performed with a variety of actors to build awareness and consensus. To facilitate this process and to develop a quality system for an organization, continuous monitoring of objectives at each stage with focus on information assurance objectives and addressing root issues associated should be addressed. For future, it is anticipated that to support this process and to integrate quality into a broad range of strategic organizations, a range of tools will be available. An unexpected finding was that qualitative information, in the form of textual descriptions of quality issues, was perceived to be the most valuable output of the review process. Cost of the implementation of this model is supposed to be high as at every stage it contains continuous monitoring, but keeping in view the nature and objectives of strategic organization, it cannot be ignored.

It is much more difficult to measure quality of a logical specification than a finished product (e.g. a working system). The conclusion from this research for data modeling practice is that a combination of "hard" and "soft" information provides the best solution to the problem of evaluating the quality of information models. Since the proposed model is a process based model laboratory analysis of high-level process based models are not feasible to provide accurate information, development of tools and methods for empirical analysis are the area that requires active research to develop tools for empirical analysis of such models.

\section{ACKNOWLEDGEMENTS}

The author acknowledges help, support and guidance provided by $\mathrm{M} / \mathrm{s}$ Oracle Pakistan, during research work at Department of Electronic Records Management System, Defence Organization. 


\section{REFERENCES}

[1] Xu, H., "Critical Factors for Accounting Information Systems Data Quality", Ph.D. Dissertation, University of Southern Queensland, 2003.

[2] Dravis, F., "Data Quality Strategy: A Step-by-Step Approach (Practice-Oriented Paper)", Proceedings of $9^{\text {th }}$ International Conference on Information Quality, 2004.

[3] Pawlowski, J.M., "The Quality Adaptation Model: Adaptation and Adoption of the Quality Standard ISO/ IEC 19796-1 for Learning, Education, and Training", Educational Technology \& Society, Volume 10, No. 2, pp. 3-16. 2007.

[4] Ehlers, U.D., "Quality in e-Learning: The Learner's Perspective", European Journal of Vocational Training. Thessaloniki, Greece, 2004.

[5] Juran, J.M., "Quality Control Handbook", McGraw-Hill, New York, NY, 1951.

[6] Cruickshank, M., "Total Quality Management in the Higher Education Sector: A Literature Review from an International and Australian Perspective", TQM and Business Excellence, Volume 14, No. 10, 2003.

[7] Ravichandran, T. "Quality Management in Systems Development: An Organizational System Perspective", MIS Quarterly, Volume 24, No. 3, pp. 381-415, 2000.

[8] Gill, N.S., "Factors Affecting Effective Software Quality Management Revisited", ACM SIGSOFT Software Engineering Notes, Volume 30, No. 2, 2005.

[9] Pipino, L.L., Lee, Y.W., and Wang, R.Y., "Data Quality Assessment", Communications of the ACM, Volume 45, No. 4, pp. 211-218, 2002.

[10] Shaha, S.H., Lewis, V.K., O’Donnell, T.J., Brown, D.H., "Evaluating Professional Development: An Approach to Verifying Program Impact on Teachers and Students", Journal of Research in Professional Learning, pp. 1-17, 2004.

[11] Sebestian-Coleman, L., "Measuring Data Quality for Ongoing Improvement: A Data Quality Assessment Framework", Book Published by Newnes, 2013.

[12] Nousak, P., and Phelps, R., "A Scorecard Approach to Improving Data Quality”, Proceedings of Data Warehousing and Enterprise Solutions, Sugi-27, Orlando, Florida, 2002.
[13] Johns, M.L., "Information Management of Health Professions", Albany, Delmar Publishers, New York, 1997.

[14] Masters, R.J., "Overcoming the Barriers to TQM's Success", Quality Progress, Volume 29, No. 5, pp. 53-55, 1996.

[15] US Department of Defense, "DoD Guidelines on Data Quality Management” 1997, (Accessible through http:/ /www.tricare.mil/ocfo/_docs/DoDGuidelines OnDataQualityManagement.pdf.

[16] Machonachy, W.V., Schou, C.D.R., Daniel, W.D., “A Model for Information Assurance: An Integrated Approach", Proceedings of IEEE Workshop on Information Assurance and Security, United States Military Academy, West Point, NY, 5-6 June 2001.

[17] Cherdantseva, Y., and Hilton, J., "A Reference Model of Information Assurance and Security", $8^{\text {th }}$ International Conference on Reliability and Security, pp. 546-555, 26 September, 2013.

[18] Thiagarajan, T., and Zairi, M., "A Review of Total Quality Management in Practice: Understanding the Fundamentals through Examples of Best Practice Applications, Part-I", The TQM Magazine, Volume 9, No. 4, pp. 270-286. 1997.

[19] Mckernan, J., "Curriculum Action Research: A Handbook of Methods and Resources for the Reflective Practitioner”, Kogan Page, London, 1991.

[20] Baker, T.L., "Doing Social Research", McGraw-Hill, 1998.

[21] Kemmis, S., and Mctaggart, R.E., "The Action Research Planner", Deakin University, Melbourne, Australia, 1988.

[22] Wynekoop, J.L., and Russo, N.L., "Studying Systems Development Methodologies: An Examination of Research Methods", Information Systems Journal, Volume 7, No. 1, January, 1997.

[23] Moody D.L., "Measuring the Quality of Data Models: An Empirical Evaluation of the Use of Quality Metrics in Practice", Ivan, et.al. (Editors) Proceedings of European Conference of Information Systems, 2003. 